The Bulletin of the Australian Mathematical Society aims at quick publication of original research in all branches of mathematics. The Editors receive more than three times as much material as can be published in the BULLETIN; many meritorious papers can, therefore, not be accepted. Authors are asked to avoid, as far as possible the use of mathematical symbols in the title. Manuscripts are accepted for review with the understanding that the same work is not concurrently submitted elsewhere.

To ensure speedy publication, editorial decisions on acceptance or otherwise are taken quickly, normally within a month of receipt of the paper. Papers are accepted only after a careful evaluation by the Editor and an Associate Editor or other expert in the field. As even minor revisions are generally not permitted, authors should read carefully all the details listed below. For a paper to be acceptable for publication, not only should it contain new and interesting results but also

(i) the exposition should be clear and attractive;

(ii) the manuscript should be in publishable form, without revision.

Authors should submit three clean, high quality copies to

The Editor, Bulletin of the Australian Mathematical Society,

Department of Mathematics, The University of Queensland,

Queensland 4072, Australia.

Unless requested at the time, material submitted to the BULLETIN will usually not be returned.

\title{
EDITORIAL POLICY
}

1. References. Arrange references alphabetically (by surname of the first author) and cite them numerically in the text. Ensure the accuracy of the references: authors' names should appear as in the work quoted. Include in the list of references only those works cited, and avoid citing works which are "in preparation" or "submitted". Where the work cited is not readily accessible (for example, a preprint) a photocopy of the title page and relevant sections of the copy that you have used will be of great help to the editors.

\section{Abstracts.}

1. Each paper must include an abstract of not more than 200 words, which should contain a brief but informative summary of the contents of the paper, but no inessential details.

2. The abstract should be self-contained, but may refer to the title.

3. Specific references (by number) to a section, proposition, equation or bibliographical item should be avoided.

3. Subject Classification. Authors should include in their papers one or more classification numbers, following the 1991 Mathematics Subject Classification. Details of this scheme can be found in each Annual Index of Mathematical Reviews.

4. Abstracts of Ph.D. Theses. The Bulletin endeavours to publish abstracts of all accepted Australasian Ph.D. theses in mathematics. One restriction, however, is that the abstract must be received by the Editor within 6 months of the degree being approved.

5. Electronic Manuscripts. The Bulletin is produced using $\mathcal{A}_{\mathcal{M}} S$ - $T_{E} X$. Authors who are able to do so are invited to prepare their manuscripts using $\mathcal{A}_{\mathcal{M}} S$-TEX. Hard copy only should be submitted for assessment, but if the paper is accepted the author will be asked to send the text on a $5 \frac{1}{4}$ IBM PC compatible diskette or via e-mail to ams@maths.uq.oz.au. [Typed manuscripts are, of course, still acceptable.] 


\section{Bulletin of the Australian Mathematical Society}

Torsion-free Abelian groups torsion over their endomorphism rings

Theodore G. Faticoni

Rings with quasi-injective ideals

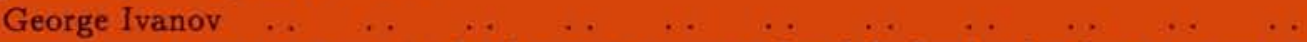

L. Coladas, Z. Li and S. Wang $\quad \begin{array}{llllllllll} & \ldots & \ldots & \ldots & \ldots & \ldots & \ldots & \ldots & \ldots\end{array}$

On strong convergence of arrays

Yong-Cheng Qi

An application of Jacobi type polynomials to irrationality measures

Ari Heimonen, Tapani Matala-aho and Keijo Väänänen _. . . . . .

Twists of matrix algebras and some subgroups of Brauer groups II

Wenchen Chi

Topological transversality: applications to differential equations

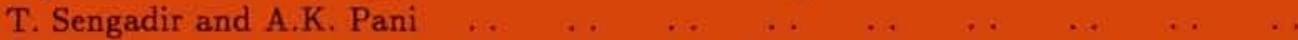

251

A Littlewood and Paley-type inequality on the ball

Jun Soo Choa and Hong Oh Kim

Méthodes fonctionnelles pour la transcendence en caractéristique finie

On prime right alternative rings with commutators in the left nucleus

Erwin Kleinfeld and Harry F. Smith

Schrödinger operators with magnetic and electric potentials

Yu Kaiqi

A note on intermediate normalising extensions

S. Dăscălescu and L. van Wyk

On some inequalities for elementary symmetric functions

Mi Lin and Neil S. Trudinger

Power roots of polynomials over arbitrary fields

Vincenzo Acciaro

Projective and affine transformations of a complex symmetric connection

Novica Blažić and Neda Bokan _. . . . . . . . .

Remarks concerning the 2-Hilbert class field of imaginary quadratic number fields: Corrigenda

Elliot Benjamin

\section{ABSTRACTS OF AUSTRALASIAN Ph.D. THESES}

Balanced block designs and various properties

Abdollah Khodkar 